\title{
Model Predictive Control of Load Transporting System on Unmanned Aerial Vehicle (UAV)
}

\author{
Aytaç Altan, Özgür Aslan, Rıfat Hacıŏlu
}

\begin{abstract}
The control system in Unmanned Aerial Vehicles (UAV), which is widely used in exploration and surveillance, target detection and tracking, remote sensing and mapping, logistics and cargo, search and rescue in dangerous areas, is very important. In this study, the model of the Load Transporting System (LTS) originally designed on UAV is obtained by linear Auto-Regressive eXogenous (ARX) model structure and the Model Predictive Control (MPC) is performed. The 4 payloads in the cubic structure can be carried by the originally designed LTS. DC servo motor is used in the LTS so that payloads can be left to the predetermined targets. When designing the MPC compared to classic PID, the limitations due to the physical properties of the LTS on the UAV are considered. The UAV autonomously flies and leaves payloads using LTS on targets.
\end{abstract}

Keywords-UAV, Load Transport Sytstem (LTS), Model Predictive Control (MPC), position control.

\section{Introduction}

The Unmanned Aerial Vehicles (UAVs), which have a great importance in today, are frequently used in military and civilian applications. UAVs that operate remotely or autonomously and landed backwards after the mission provide convenience in many ways. UAVs that can be loaded onto the main body of useful loads have been used both in civilian and military areas in recent years. UAVs perform many different missions, including search and rescue operations, surveillance, inspection, mapping [1], load transport, aerial photographing and law enforcement. UAVs are quite useful when these tasks are performed in hazardous and inaccessible environments. UAVs contain many engineering challenges in the fields of electrical, mechanical and control engineering [2-4].

Aytaç Altan

Bülent Ecevit University

Turkey

Özgür Aslan

Bülent Ecevit University

Turkey

Rıfat Hacıoğlu

Bülent Ecevit University

Turkey
In order to perform UAVs assigned tasks effectively in a short period of time, it is necessary to apply effective control algorithm as in the flight control system [5], the camera gimbal system [6], the Vertical Take-Off and Landing (VTOL) system [7] and the Load Transporting System (LTS) [8-10]. In this study, the model of the LTS originally designed on UAV is obtained by linear autoregressive exogenous (ARX) model structure and the Model Predictive Control (MPC) is performed.

There are many studies in the literature on the control of UAVs for different types of load transporting. In most of the studies carried out, the loads are carried on the UAV's body as hanging. In [10], authors proposes a nonlinear control strategy to solve the suspended load Transportation problem using a Tilt-rotor UAV. The authors aim to keep both the UAV and the load stable throughout the whole trajectory in this study, even in the presence of parametric uncertainties and measurement errors. In [11], the effect of dynamic load disturbances caused by suddenly increasing load mass is investigated and how those affect the UAV under PID flight control. The dynamics and control of the UAV which transporting a payload connected by a flexible cable, modeled as a serially-connected link system, is expressed in [12], Also in [13], an Interconnection and Damping AssignmentPassivity Based Control (IDA-PBC) is developed for a UAV that transports a cable suspended payload is developed. The authors are pointed out that the control law does not depend on the cable's swing angle.

In this study, in order to leave a load on targets previously determined by the LTS on the autonomously flying UAV, the model of the originally designed LTS on the UAV is obtained by the linear ARX structure and the MPC is performed. The 4 payloads in the cubic structure can be carried by the originally designed load carrying system. The MPC and PID control of the DC servo motor used in LTS is carried out so that the payloads can be left to the predetermined targets. When designing the MPC compared to classic PID, the limitations due to the physical properties of the LTS on the UAV are considered.

This article is organized as follows: the design and modelling of LTS on UAV is described in Section II. The MPC of LTS on UAV is expressed in Section III. Simulation and experimental results are presented in Section IV. Conclusions are finally given in Section V. 


\section{Load Transporting System Design and Modeling}

Load transportation is one of the many applications of interest for UAV. UAVs which have load transporting systems are currently in use to provide logistical support, especially in search-rescue and military operations, to designated regions in the operation area. This section focuses on the original LTS design and the designed LTS model. In this study, six motor UAV (hexacopter) is used and the design of the LTS is carried out in accordance with the hexacopter.

\section{A. LTS Design}

LTS is designed to leave four payloads to the predetermined targets. The UAV moves autonomously and the LTS's load-transporting to predetermined targets is also performed autonomously. DC servo motor is used in the LTS so that payloads can be left to the predetermined targets. The front and side view of the designed LTS in simulated environment are shown in Figure 1, and the front and side view of the realized LTS on the hexacopter are shown in Figure 2 and Figure 3, respectively.
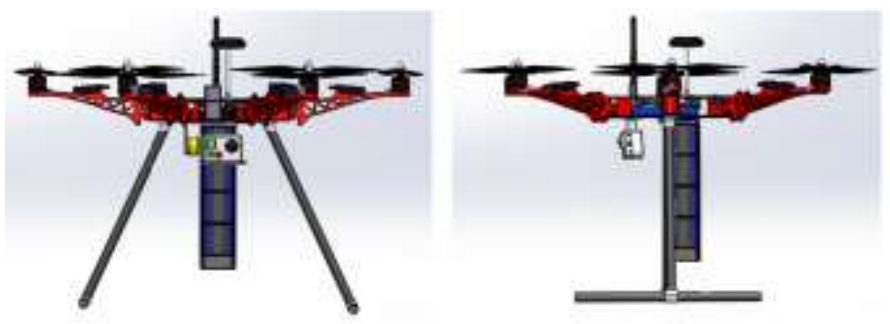

Figure 1. Front and side view of designed LTS.

The LTS is mounted perpendicular to the lower surface of the UAV body so that the swing can be minimized. Thus, it is ensured that the UAV follows the predetermined route with high performance since the swing is reduced.

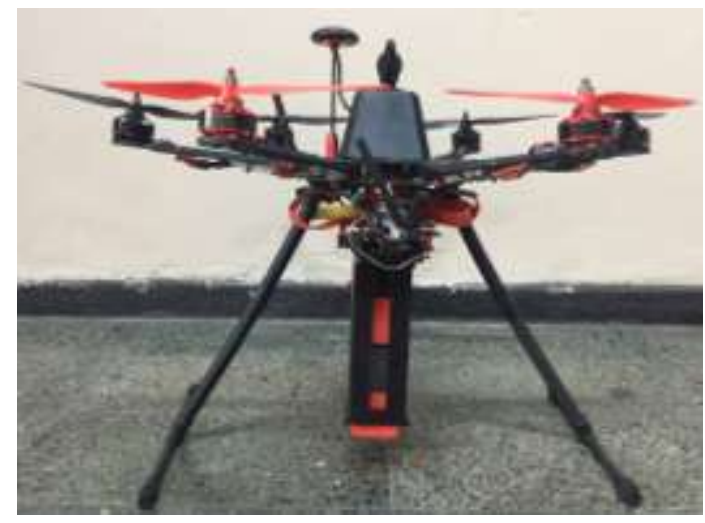

Figure 2. Front view of realized LTS on hexacopter.

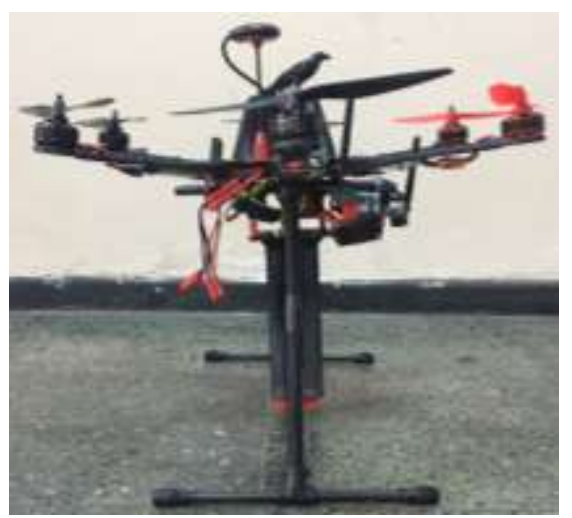

Figure 3. Side view of realized LTS on hexacopter.

\section{B. Identification ARX Model for LTS}

System identification is a method of obtaining mathematical model in a dynamic system based on input/output data [14]. Modeling is important when system gain and system dynamic behavior need to be determined. Discrete time system models that sampled data is frequently used. The purpose of system identification can be defined as "find a model with adjustable parameters and then set these parameters to match the predicted output with the measured output" [15]. The most important part of the MPC design process is to obtain the appropriate mathematical model of the system. In this study, discrete time linear ARX model structure is realized together with system identification problem.

Many researchers evaluate system dynamics in a linear or nonlinear structure with black box, gray box, or physical modeling based on system pre-information. There are many structures among the commonly used parametric model structures for linear dynamic systems. Such as ARX, AutoRegressive Moving Average with eXogeneous (ARMAX), Auto Regressive Moving Average (ARMA), Box-Jenkins (BJ) and Output Error (OE) [14]. In parameter estimation, basic standard and statistical techniques such as least squares, recursive least squares and cumulative least squares methods are used [16].

The systems defined by the ARX model structure have linear properties [14] thus LTS which used in study can be defined using ARX model structure. The block diagram of single input single output (SISO) linear system in Figure 4:

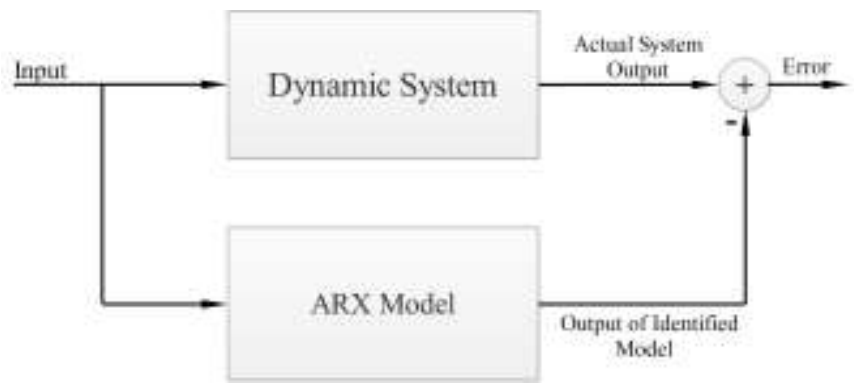

Figure 4. ARX model aproach of SISO linear system. 
The transfer function is expressed by ARX model such as

$$
H\left(q^{-1}\right)=\frac{\sum_{k=0}^{M} b_{k} q^{-k}}{1+\sum_{k=1}^{N} a_{k} q^{-k}}
$$

where $q^{-1}$ is the delay operator, $a_{k}$ and $b_{k}$ are denominator and numerator polynomial coefficients, respectively. The output, $y(n)$ obtained depending on the input, $x(n)$ as follows:

$$
y(n)=-\sum_{k=1}^{N} a_{k} y(n-k)+\sum_{k=0}^{M} b_{k} x(n-k)
$$

In this study, ARX model structure parameters $a_{k}$ and $b_{k}$ are obtained by least squares method. Here it is minimized error between actual system output and output of the identified model for given same input in the least squared sense as seen in Figure 4.

\section{Model Predictive Control (MPC) of LTS}

This section describes the control strategy design for LTS. The MPC is an optimization algorithm that uses system models to examine the responses of the system to future inputs. The MPC algorithm is optimized the system's future behavior at every control interval. MPC is computed the future input/output of the process using the system model and instant measurements in the system. MPC is defined as the minimization of the cost function that defines the problem [17]:

$$
J=\sum_{k=1}^{p} \lambda_{k}(r(n+k)-y(n+k \mid n))^{2}+\sum_{k=1}^{q} \delta_{k} \Delta u(n+k \mid n)^{2}
$$

where $p$ is prediction horizon, $q$ is control horizon, $\lambda_{k}$ and $\delta_{k}$ are weight coefficient of system output and weight coefficient that determines the effect of $\Delta u$, respectively. In this study, the values minimizing (3) are computed and the output signal of the MPC, which is the control signal of LTS is generated using the system model.

\section{Iv. Simulation and Experimental Results}

In this study, experimentally obtained data sets of LTS, expressed to as SISO, on the hexacopter in Figure 2 were used. The hexacopter was moved autonomously in the environment with a wind speed of $4.8 \mathrm{~m} / \mathrm{s}$. The wind with 4.8 $\mathrm{m} / \mathrm{s}$ speed was evaluated as external disturbance effect and data sets obtained under external disturbance effect were used in the study. The model parameters of the system are estimated by least squares method. The MSE value between the system output values in the used data set and the output values of the estimated model is calculated as 0.462 . Using the data sets, the ARX model of LTS was obtained with $97.78 \%$ performance and was shown in Figure 5.

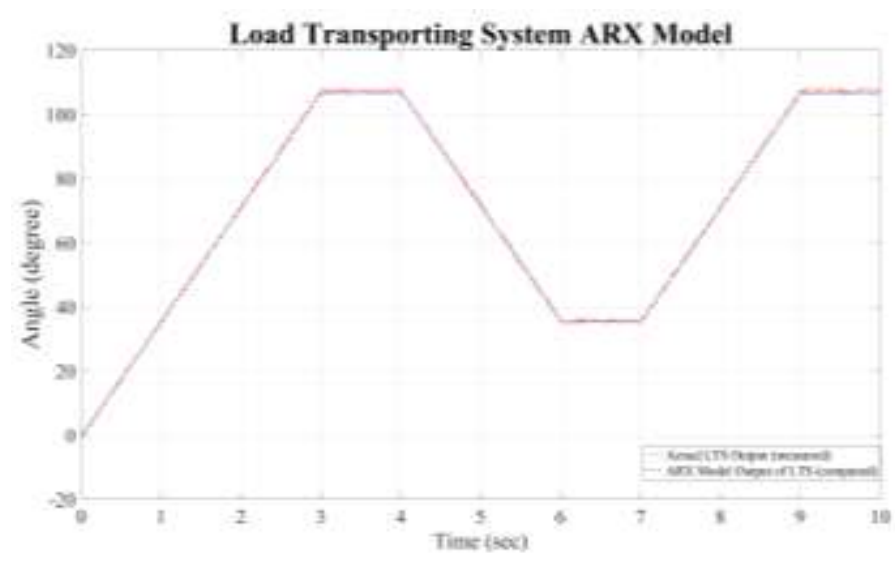

Figure 5. ARX model of LTS.

The transfer function obtained is shown as follows:

$H(q)=\frac{0.4427 q^{-1}-0.09015 q^{-2}}{1-0.4927 q^{-1}-0.5073 q^{-2}}$

The position control of the ARX model of the LTS is made with MPC. In the designed controller, the prediction horizon is set to 15 and the control horizon is set to 5. In addition, The LTS is also controlled by PID. The coefficients of the PID controller were determined as $K_{p}=0.15149, K_{i}=0.1317$ and $K_{d}=0.0072452$. The MPC and PID results of LTS are shown in Figure 6.

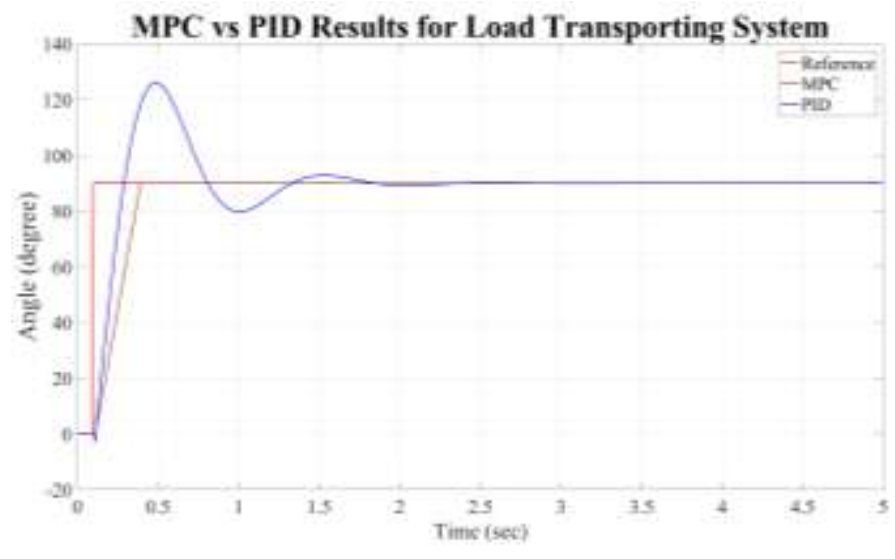

Figure 6. MPC vs PID Results for LTS.

Figure 6 shows that the performance of the MPC according to the PID is visibly better. The settling time of the MPC is about $0.35 \mathrm{sec}$ while the settling time of the PID controller is about $2.9 \mathrm{sec}$. In the study, the rising time $\left(t_{r}\right)$ and the overshoot $\left(M_{p}\right)$ of the PID controller were determined as 0.3 sec and $38.9 \%$, respectively. With the LTS on the UAV, 4 payloads have been left to predetermined targets with both MPC and PID control. In the tests, it was observed that payloads were carried with high performance to predetermined targets. 
Proc. of the Fifth International Conference on Advances in Mechanical and Robotics Engineering - AMRE 2017. Copyright (C) Institute of Research Engineers and Doctors. All rights reserved.

ISBN: 978-1-63248-123-8 doi: 10.15224/ 978-1-63248-123-8-14

\section{v. Conclusion}

The LTS was originally designed to reduce the swing to the minimum. The linear second order ARX model of the system using the input-output data experimentally obtained under the external disturbance effect of the LTS on the autonomously moving UAV was obtained. The control of the LTS on the autonomously moving UAV was made with PID and MPC. The obtained PID and MPC results were compared. With the MPC controlled LTS, 4 payloads in the cubic structure have been transported to predetermined targets. When designing the MPC compared to classic PID, the limitations due to the physical properties of the LTS on the UAV were considered. It has been seen that the performance of the MPC according to PID is visibly better.

\section{Acknowledgment}

This study was supported by Bülent Ecevit University (BAP Project No: 2014-75737790-01). The authors would like to thank Bülent Ecevit University for their support.

\section{References}

[1] A. Altan, K. Bayraktar, and R. Hacıoğlu, "Simultaneous localization and mapping of mines with unmanned aerial vehicle," IEEE 24th Sig. Proc. and Comm. Appl. Conf., pp. 1433-1436, May 2016.

[2] J. J. Xiong and E. H. Zheng "Position and attitude tracking control for a quadrotor UAV," ISA Trans., vol. 53(3), pp. 725-731, May 2014.

[3] L. Luque-Vega, B. Castillo-Toledo, and A. G. Loukianov, "Robust block second order sliding mode control for a quadrotor," J. Frankl. Inst., vol. 349(2), pp. 719-739, March 2012.

[4] L. Derafa, A. Benallegue, and L. Fridman, "Super twisting control algorithm for the attitude tracking of a four rotors UAV," J. Frankl. Inst., vol. 349(2), pp. 685-699, March 2012.

[5] K. Alexis, C. Papachristos, R. Siegwart, and A. Tzes, "Robust model predictive flight control of unmanned rotorcrafts," J. Intel.\&Robotic Sys., vol. 81(3), pp. 443-469, March 2016.

[6] A. Altan and R. Hacioğlu, "The controller of the camera used in target tracking for unmanned vehicle with model predictive controller," IEEE 22nd Sig. Proc. and Comm. Appl. Conf., pp. 1686-1689, April 2014.

[7] G. S. Hadi, M. R. Kusnaedi, P. Dewi, A. Budiyarto, and A. Budiyono, "Design of avionics system and control scenario of small hybrid vertical take-off and landing (VTOL) UAV," J. Instr., Automation and Sys., vol. 2(2), pp. 66-71, 2015.

[8] F. A. Goodarzi, D. Lee, and T. Lee, "Geometric control of a quadrotor UAV transporting a payload connected via flexible cable," Int. J. C., Automation and Sys., vol. 13(6), pp. 1486-1498, 2015.

[9] B. S. Rego and G. V. Raffo, "Suspended load path tracking by a tiltrotor UAV," IFAC-PapersOnLine, vol. 49(32), pp. 234-239, 2016.

[10] M. M. de Almeida and G. V. Raffo, "Nonlinear control of a tiltrotor UAV for load transportation," IFAC-PapersOnLine, vol. 48(19), pp. 232-237, 2015.

[11] P. E. I. Pounds, D. R. Bersak, and A. M. Dollar, "Stability of small-scale UAV helicopters and quadrotors with added payload mass under PID control," Auton. Robot., vol. 33, pp. 129-142, 2012.

[12] F. A. Goodarzi, D. Lee, and T. Lee, "Geometric stabilization of a quadrotor UAV with a payload connected by flexible cable," American C. Conf. (ACC), pp. 4925-4930, June 2014.
[13] M. E. Guerrero, D. A. Mercado, R. Lozano, and C. D. Garcia, "Passivity based control for a quadrotor UAV transporting a cable-suspended payload with minimum swing," IEEE 54th Ann. Conf. D. and C. (CDC), pp. 6718-6723, December 2015.

[14] L. Ljung, System Identification: Theory for the User. Prentice Hall, New Jersey, 1999.

[15] N. Taghavi and A. Sadr, "Piezoelectric transducer modeling: with system identification (SI) method," World Academy of Sc., Eng. and Tech., pp. 296-301, 2008.

[16] G. Wang, T. Li, G. Zhang, X. Gui, and D. Xu, "Position estimation error reduction using recursive-least-square adaptive filter for model-based sensorless interior permanent-magnet synchronous motor drives," IEEE Trans. Ind. Elect., vol. 61(9), pp. 5115-5125, September 2014.

[17] E. F. Camacho and C. Bordons, Model Predictive Control. Springer Science\&Business Media, 2013.

About Authors:

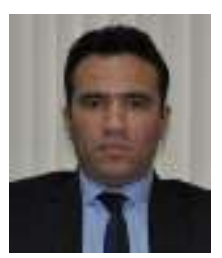

Aytaç Altan is a lecturer at the department of Electrical Electronics Engineering, Bulent Ecevit University in Turkey. He received the BSc and M.Sc. degrees in the department of Electrical Electronics Engineering from Anadolu University in 2011 and 2016, respectively. He is a Ph.D. candidate. His research interests are robotic systems, adaptive control and system identification.

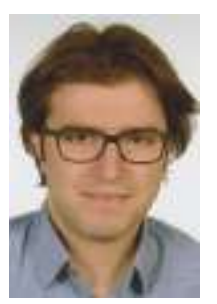

Özgür Aslan works as an automation engineer at Erdemir Iron and Steel factory. He received the BSc and M.Sc. degrees in the department of Electrical Electronics Engineering from Zonguldak Karaelmas University in 2006 and 2009, respectively. $\mathrm{He}$ is a Ph.D. candidate at BEU. His research interests are automation systems, adaptive control, fuzzy logic and system identification.

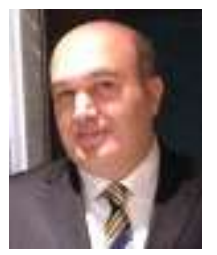

Rıfat Hacıoğlu is an Assistant Professor of department of Electrical Electronics Engineering at the Bulent Ecevit University in Turkey. He received the M.Sc. and Ph.D. degrees in the department of Electrical Electronics Engineering from Illinois Institute of Technology in 1996 and 2002, respectively. His research interests are signal processing, control applications, linear and nonlinear system identification, parametric model estimation approaches, microprocessor based control systems, image identification problems. 